\title{
A Table Tennis Game for Three Players
}

\author{
Florian 'Floyd' Mueller \\ The Department of Information Systems \\ The University of Melbourne \\ Parkville, VIC 3010, Australia \\ floyd@exertioninterfaces.com
}

\author{
Martin Gibbs \\ The Department of Information Systems \\ The University of Melbourne \\ Parkville, VIC 3010, Australia \\ martinrg@unimelb.edu.au
}

\begin{abstract}
Table tennis is a game that can provide healthy exercise and is also a social pastime for players of all ages across the world. However, players have to be collocated to play, and three players cannot usually play at the same time in fair or equitable manner. We have developed a networked table tennis like game called Table Tennis for Three (TTT). TTT is a game played with bat and ball by three people on three physically separated table tennis tables. The players of TTT can interact with one another through the use of augmented virtuality - the augmentation of virtual systems with elements of physical game play. TTT uses the physicality of table tennis combined with the communicative media typically associated with videoconferencing. TTT has been developed with the aim of achieving similar benefits to those of co-located table tennis such as exercise, enjoyment and bringing people together to socialize.
\end{abstract}

\section{Author Keywords}

Exertion interface, table tennis, ping-pong, sports, physical interaction, social interaction, tangible interface, augmented reality, augmented virtuality.

\section{ACM Classification Keywords}

H5.2. Information Interfaces and presentation (e.g., HCI): User Interfaces.

\section{INTRODUCTION}

Email, instant messenger, mobile phone text messaging and the like are typically derived from a task-oriented world, initially designed to improve efficiency in a work environment. However, people have appropriated these telecommunication technologies for sociable ends; to help satisfy the need to create and foster social bonds. If we take a sociability approach to the design of technology, what would an interface look like that was designed, from the outset, to support social interactions?

Furthermore, the initial design goals many telecommunication tools seems to have been to emulate the co-located experience as much as possible; recent developments often focus on the increase of audio quality and video resolution in videoconferencing systems in order to create a "better than being there" experience.

OzCHI 2006, November 22-24, 2006, Sydney, Australia.

Copyright the author(s) and CHISIG

Additional copies are available at the ACM Digital Library

(http://portal.acm.org/dl.cfm) or ordered from the CHISIG secretary

(secretary@ chisig.org)

OZCHI 2006 Proceedings ISBN: 1-59593-545-2
However, some researchers in telepresence have suggested that it may be beneficial to aim to "be different" and let go of the paradigm of trying to accurately reproduce the real world (Hollan and Stornetta, 1992). Seeking a different experience could be more rewarding, and the distribution of the users can provide opportunities, not available in a co-located setting, which technology can exploit.

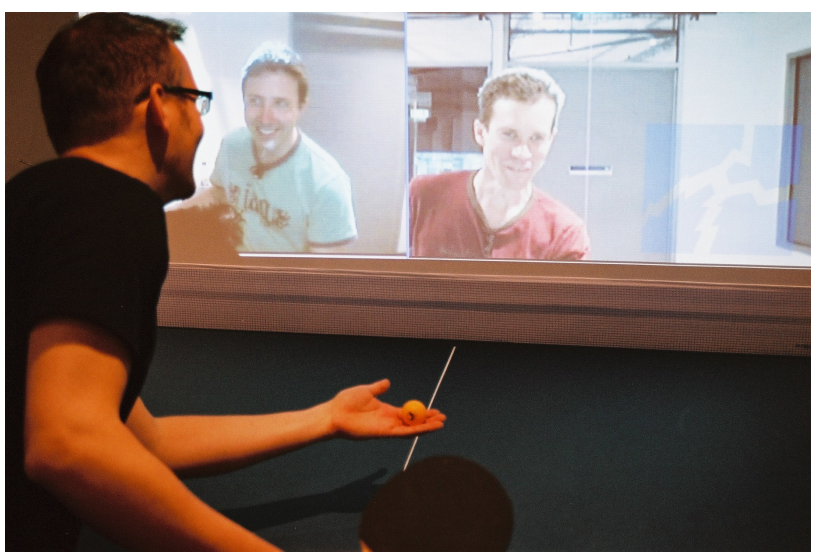

Figure 1 Table Tennis for Three

Table Tennis for Three (Figure 1) is an attempt to respond to these issues and challenges through augmented virtuality. In Table Tennis for Three we have augmented a virtual video conferencing system with elements of physical game play from table tennis. We have focused our design on these two points:

- We were interested in how an interface would look like if designed to support social interactions between geographically distant participants from the outset.

- We aimed for a demonstrator to show that if users are in different locations it can be advantageous for the experience rather than being a problem that networking technologies need to fix.

We have therefore designed a table tennis game that, similar to traditional table tennis, supports social interactions between players, but can additionally be played by players who are geographically apart. Furthermore, three players can play the game simultaneously, a situation that is not possible if the players are co-located.

\section{TABLE TENNIS FOR THREE}

The validity of using physical interfaces to promote a sociable interaction has been demonstrated by previous work on "Exertion Interfaces" and the use of competitive sport for promoting rapport between video conference participants (Mueller et. al. 2003). Previous research 
suggests that rich interaction between participants in online games can be enhanced by providing additional means of communication to players beyond the keyboardmouse, joystick or game pad interfaces typically used in these games (Gibbs et. al. 2004). Thus, we believe, the interface for a game intended to promote sociable interaction needs to move beyond the current mousekeyboard interaction paradigm.

We have therefore built a demonstrator of a sport over a distance, hoping that the social benefits of table tennis can be achieved when executed in a network environment, with a focus on a real physical interface instead of a virtual keypad experience, as demonstrated by "Breakout for Two" (Mueller et. al. 2002). We use a very similar game play for the table tennis system, but extend it to three geographically distant locations.

The players of Table Tennis for Three are able to talk and see each other at all times, while being simultaneously engaged in a ubiquitous environment which includes audio and visual data in a video conferencing system augmented with elements of physical game play. Our prototype uses the physicality of table tennis combined with the communicative media associated with videoconferencing. Table Tennis for Three enables competition and conversation between three participants who are all distantly located from one another.

\section{RELATED WORK}

Systems that use an exertion interface to deliver a physical leisure or sports experience over a network to support social interactions have increasingly appeared over the last couple of years, and we might see more commercially available in the not so distant future (Marriott 2004; Cooper 2004).

Telephonic Arm Wrestling is an early example (built in 1986) of a networked exertion interface, although it did not seem to have worked particularly well, allowing both players to win under some circumstances (Shanken 2000). Dance Dance Revolution Ultramix (Dance Dance Revolution Ultramix 2005) is a home version of the popular exerting arcade game, in which the players follow dance instructions on the screen with their feet on touch sensitive tiles. It can be very exhausting, but also quite social, drawing large crowds when good dancers "enter the stage". Virku is a Virtual Fitness Centre that uses physical movements conducted on exercise bicycles to modify the representation of a 3D virtual environment (Mokka et. al. 2003). PingPongPlus (Ishii et. al. 1999) is an augmented table tennis table with a projection that reacts to the impact of the ball; however, it supports only two co-located players. Airhockey over a Distance is an airhockey game that is playable by distributed players by using a physical puck that is shot out at the other end by puck cannons whenever the player hits the puck across the middle line (Mueller et. al., 2006). Push'N'Pull is a networked exercise machine, which the players use as interface for a cooperative game, supported by a highdefinition videoconference (Mueller et. al., 2006). Virtual Tug-of-War was an exhibition at the New York Hall of Science. Visitors could pull on a rope attached to a calibrated pulley and compete with others using similar equipment at another site 13 miles away. Breakout for Two (Mueller et. al. 2003) is an exertion interface that allows two players to play a soccer-like game together, communicating via a body-size videoconference.

Although these examples allow players to experience a physical and sometimes social experience despite being geographically distant, they usually support only two players simultaneously. Furthermore, the depicted systems try to recreate and emulate the co-located experience, but do not leverage the network component as a unique opportunity to achieve something that is not possible if all participants are in the same room.

\section{THE GAMEPLAY OF TABLE TENNIS FOR THREE}

Each player has one ball, a bat and a table tennis table. The table is set-up so that the ball can be hit against the vertically positioned opposite half of their table. This setup is familiar to table tennis players who practice on their own by playing the ball against the board.

The vertical part of the table is painted white to also serve as projection surface for a videoconference of the other players. On the left is the video of player 1 , on the right player 2. Projected on top of the videoconference are eight semi-transparent targets that players have to hit with their ball. These targets, or bricks, "break" when hit by players. The bricks are synchronized across the three tables, so the other players see the same brick layout and the same brick states. If a brick is hit once, it cracks a little. If it is hit again (regardless by which player), it cracks more. If hit three times, it "breaks" and disappears, revealing the underlying videoconferencing completely: the player "broke" through to the remote players. However, only the player that hits the brick the final time and makes it disappear receives the point. This adds an element of interactive competition to the game: players can try to snatch away points by hitting bricks that have been already hit twice by the other players.

We chose this approach to reflect a characteristic of traditional table tennis, where one player's actions depend on the other player's actions. Unlike with networked exercise machines, where each participant works out individually and compares scores at the end, this approach can accommodate different strategies during game play, based on what the opponent does, in order to outsmart him or her.

Each broken brick scores one point, and the current score is display on top of the board. Once all bricks are cleared, the scores are compared and the player with the most points wins the game.

\section{WHY TABLE TENNIS}

We are aiming to demonstrate that it is possible to experience a social and physical leisure activity across several geographically distinct locations. We based our system based on table tennis for a number of reasons.

First, table tennis is often considered a social activity. Table tennis tables can be found in social spaces such as community centres, schools, social clubs, and so forth. 
Table tennis attracts players of all ages, is one of the most popular sports in the world in terms of player numbers (Wikipedia 2006), and has very simple rules that allow everyone to pick up the game within minutes. The game's ability to engage people quickly can serve as a catalyst for interpersonal communication and facilitate rapport. A game can help participants loosen-up, serve as an icebreaker, and exhaust us physically. Such social sports activities have been described as a potentially rewarding way to bring people together (Nauright, 2003). They also hold the potential to facilitate team-building by contributing to the tacit and shared development of trust, mutual awareness, and shared social skills.

Second, table tennis is a sport as well as a social activity. Table tennis is an Olympic discipline, and professional leagues exist around the world. Unlike other sports - such as rowing, equestrian or pole vault - table tennis with its simple rules, quick sense of achievement and affordable setup caters for casual players as well as professionals. Wikipedia (2006) suggests to distinguish between social table tennis and professional table tennis by calling the recreational activity ping-pong.

Third, table tennis is easy to set-up. It does not require a large area such as tennis, the table and the net are relatively inexpensive, and paddles are available in many stores. Many people have a table in their garage for casual games when friends come over, for example.

Fourth, table tennis is competitive, even when played on a recreational level. Unlike other social physical activity, such as dancing, table tennis has a competitive scoring scheme; there is no collaborative version of the game. This emphasises the sports character of the game, and we believe the competitiveness is an important aspect of a successful implementation of a networked sports activity.

Playing table tennis, or any other leisure sport, can offer a means to connect with others socially. Engaging in sports is an intriguing way to interlock bodily physics and cultural meaning (Steinkamp, 1983). We believe table tennis with the described characteristic is a very suitable example for our research agenda, but other sports might also be appropriate.

\section{WHY THREE PLAYERS?}

Instead of trying to most accurately recreate the local experience of playing sports together in a distributed setting, one of our aims is to create an experience that is only possible because of the distance between the participants: a game of table-tennis for three. If collocated, only two or four players can play a game of table tennis together; if three players want to play, they would have to split up unequally: one against two, creating a biased game, or adopting some kind of roundrobin system for play. Allowing three players to play a game simultaneously poses some interesting questions: How does this setup influence the interactions between the players? Is the experience comparable to having two players or two teams? Do the benefits of sports activity apply to this three-way game concept equally well?
Usually, transferring established collocated sports experiences to a distributed setup creates various challenges, often associated with bandwidth, delay and immersiveness. The physical distance between the players is predominately regarded as hindrance or challenge, but rarely as an opportunity. However, we propose to see this physical distance as an opportunity to create a novel sports experience. Our game not only allows for, but requires the players to be geographically distant, while simultaneously aims to facilitate the benefits of collocated sports, such as the physical activity and the social rapport between the players.

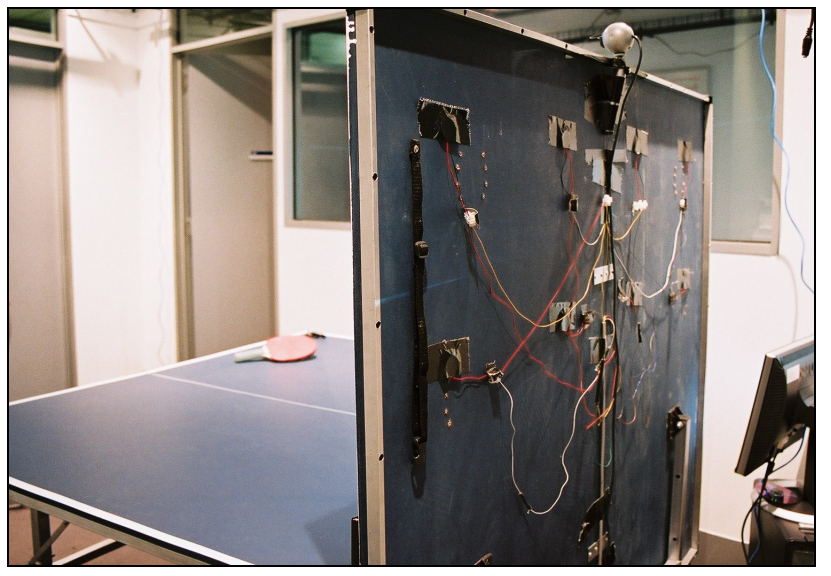

Figure 2 Sensors on the back of the table

\section{TECHNICAL SETUP}

We have built a distributed setup for Table Tennis for Three: three table tennis tables are placed in three different locations and are connected by an IP network, which supports a high-resolution audio- and videoconference. The video streams of the remote players are projected onto the vertical other half of the table surfaces. The audio is transmitted via Bluetooth headsets along with the videoconference over the network.

A ball's impact on the vertical part of the table is detected with an array of piezoelectric sensors attached to the back of the board (Figure 2). They are triggered by vibrations that travel through the wood of the table following the ball's impact. The sensor that receives the first signal to exceed a threshold value determines the location of the impact and delivers it to software that updates all other stations using client-server architecture. Each station then updates the graphical content accordingly, and synchronizes game data such as the score.

Currently, the game has only been tested on a local area network due to the bandwidth requirements of the videoconferencing, but the software is designed to accommodate bandwidth changes, so a deployment via the Internet should be feasible.

\section{EVALUATION}

We are planning to evaluate the system quantitatively and qualitatively: After having played Table Tennis for Three, we will ask participants for their opinions through a questionnaire. We will also interview them to gain 
additional insights into their experience and collect suggestions for improvement to the system.

We will evaluate the usability of the game in order to optimize the system for ease of play and to enhance the game's ability to mediate social interactions. We will investigate whether the game can mediate sociable interaction and if players develop a sense of rapport or mutual presence through game play. Playability evaluations will be used to further develop the rules of play that will produce the most fun and engaging game.

This evaluation will serve to validate whether or not a networked game with an engaging rich exertion interface is a suitable template for the design of games intended to support social connectedness between remote participants. It can also demonstrate that the distribution of players can be advantageous in a physical game, because the network opens up an opportunity, instead of solely bridging the gap between geographical separate locations.

\section{FUTURE WORK}

Initially, we are planning on letting participants experience Table Tennis for Three and collecting their impressions, as described above. Following these evaluation we hope to develop a second prototype that refines the usability and playability of the game system. This redeveloped version of Table Tennis for Three will be used to further explore our hypotheses by placing it in public and semi-public spaces such as office tea rooms and art galleries and allow participants to walk-up and play the distributed game. Using qualitative research methods, we will focus on evaluating the sociability of the game for both occasional play and for those who have the opportunity to play it repeatedly over periods of several weeks. We will evaluate the sociable effects it may have on the players, and will evaluate if they encountered an increased sense of presence or rapport through game play.

\section{CONCLUSION}

Table Tennis for Three is a novel networked table tennis game that uses real, physical balls (in contrast to virtual) to allow geographically distant players to play competitively against each other. It features an Exertion Interface in order to support a sense of social rapport between players, facilitated through an augmented videoconference. Table Tennis for Three leverages the networking component to enable something that is not possible if the players are collocated: three players can play table tennis simultaneously.

\section{ACKNOWLEDGMENTS}

The work reported in this paper was initially supported by The University of Melbourne and CSIRO Collaborative Research Support Scheme. We would like to thank Shannon O'Brien, Matt Adcock, Bo Kampmann Walther and Keith Gibbs for their help and advice on the project.
We would also like to thank the IDEAS Lab and the Interaction Design Group at The University of Melbourne for their support. We would especially like to thank Ivo Widjaja for his assistance and support.

\section{REFERENCES}

Cooper, S. Get Physical! Entrepreneur.com. (2004) http://www.entrepreneur.com/Magazines/Copy_of_MA _SegArticle/0,4453,316342,00.html

Dance Dance Revolution Ultramix (2005). http://www.xbox.com/en-US/ddr/default.htm

Gibbs, M.R., Hew, K. and Wadley, G. Social Translucence of the Xbox Live Voice Channel. In Proc. ICEC 2004. Springer-Verlag (2004), 377-385.

Hollan, J. and Stornetta, S. Beyond Being There. In Proc. CHI 1992, ACM Press (1992), 119-125.

Ishii, H., Wisneski, C., Orbanes, J., Chun, B., and Paradiso, J. PingPongPlus: Design of an AthleticTangible Interface for Computer-Supported Cooperative Play. In Proc. CHI 1999, ACM Press (1999), 394-401.

Marriott, M.: Long-Distance Sports. The New York Times, Technology. 26 Aug (2004).

Mokka S., Väätänen A., Välkkynen P. Fitness Computer Games with a Bodily User Interface. In Proc. ICEC 2003. ACM Press, (2003), pp 1-3.

Mueller, F., Agamanolis, S., Picard, R.: Exertion Interfaces for Sports over a Distance. In UIST 2002 ACM Symposium on User Interface Software and Technology. ACM Press, New York (2002).

Mueller, F., Agamanolis, S., Picard, R.: Exertion Interfaces: Sports over a Distance for Social Bonding and Fun. In Proc. CHI 2003. ACM Press, USA (2003), 561-568.

Mueller, F., Cole, L., O'Brien, S., Walmink, W. Airhockey Over a Distance - A Networked Physical Game to Support Social Interactions. In Proc. of Advances in Computer Entertainment Technology (ACE 2006), ACM Press, (2006).

Mueller, F., Stevens, G., Thorogood, A., O'Brien, S., Wulf, V. Sports over a Distance. Journal of Personal and Ubiquitous Computing, Special Issue on Movement Based Interaction (2006).

Nauright, J.: Nostalgia, Culture and Modern Sport. In Verner Møller and John Nauright (eds.): The Essence of Sport, Odense, University Press of Southern Denmark (2003).

Steinkamp, E.: Was ist eigentlich Sport? Wuppertal: Hans Putty, Germany (1983).

Shanken, E.A. Tele-Agency: Telematics, Telerobotics, and the Art of Meaning. Art Journal, Summer, 2000.

Wikipedia, Table tennis, http://en.wikipedia.org/w/ index.php?title $=$ Table_tennis\&oldid $=73275664$ 